\title{
Energy Efficient Data Aggregation using Voronoi based Genetic Clustering Algorithm in WSN
}

\author{
S. Nithyakalyani \\ Assistant professor \\ K.S.R College of Engineering \\ Tiruchengode, India
}

\author{
S. Suresh Kumar, Ph.D \\ Principal \\ Vivekanandha College of Technology for Women \\ Tiruchengode, India
}

\begin{abstract}
Wireless Sensor Network is an major emerging technique in wireless communication technology for application across a wide array of domains such as the military surveillance, medical diagnosis, weather forecasting, fire detection alarming systems, etc. One of the main challenges of wireless sensor network (WSN) is how to improve its time of livelihood due to the restricted energy of sensor nodes. Data must be aggregated in order to avoid amounts of traffic in the network, limit the recourses and energy. To solve the above dilemmas, data mining process such as clustering and data aggregation is used .clustering is used to group the nodes where as data aggregation function like MIN,MAX,AVG is used for swabbing redundant data transmission and improves the life span of energy in wireless sensor network. In this paper a new approach related to Voronoi based Genetic clustering (VBGC) Algorithm is proposed for energy efficient data aggregation. Our algorithm achieves energy efficiency by reducing the number of data transmission in each round to cluster head and from it to Base station (BS) .The Base Station periodically executes the proposed algorithm to select new Cluster-Heads after a certain period of time. Simulation results reveal that our algorithm outperforms basic GA.
\end{abstract}

\section{Keywords}

Wireless Sensor Networks, Voronoi Diagram, Genetic Algorithm, clustering, data aggregation

\section{INTRODUCTION}

Wireless Sensor Networks (WSNs) is essential ingredient of many application environments that are used in military and civilians. A sensor network is composed of a large number of sensor nodes, which are densely deployed either inside the phenomenon or very close to it [1]. One of the most important features in WSNs belongs to the limited battery of sensor nodes. When battery-powered wireless sensor nodes are placed in a specific field, it is complex to replace their batteries or provide additional energy. Furthermore, if one sensor node consumes completely its energy, part of the network may disconnect [2].

Data gathering is a common but it is significant operation in many applications of WSNs, while data aggregation and hierarchical mechanism are widely used techniques. Data aggregation can eliminate data redundancy and reduce communication load. Data mining technique like clustering mechanisms is an effective means for running such high population of nodes and can help reduce the nodes' energy consumption [3]. Some efforts in energy-efficient clustering are as follows. [2] have proposed a so-called Low Energy Adaptive Clustering Hierarchy (LEACH) as the first clustering protocol. In this protocol, a data collection area is randomly divided into several clusters while each cluster has a cluster-head and some cluster members. Cluster members transmit data to their own cluster-heads, which aggregate and transmit data to the base station. Later, [4] extended their work and represented a LEACH-C protocol. In this clustering method, the base station makes sure that only nodes with enough energy are participating in.

A protocol named HEED has been proposed by [5]. The protocol selects cluster-heads through $\mathrm{O}(1)$ time iteration according to some metrics and adopts the multi-hop communication to reduce energy consumption. The transmission cost in WSNs is regarded as the energy consumption of the nodes and when we can minimize this value, energy is saved. Fig. 1 shows a data delivery example in a cluster based network [1]. As shown, the calculation of transmission cost can be divided into two parts. The first part is the cluster members that transmit data to their cluster-heads. The second part, however, is the cluster-heads that transmit aggregated data to a sink node.

The Genetic Algorithm (GA) is a technique for randomized search and optimization and has been applied to a wide range of studies [6,7]. A basic operation flow of GA includes creating initial population, evaluating fitness, selection, crossover, mutation, updating optimal chromosome, and checking termination condition.

Searching for optimal clusters can be done using GA. Following, are the energy-efficient clustering technique using GA that has been developed previously. Electing a clusterhead that can minimize the maximum intra-cluster distance between itself and its cluster members by optimize energy consumption of the network have been proposed in [8]

The researcher in [9] have developed a GA that creates energy efficient clusters for routing in wireless sensor networks. For evaluate the energy consumption of the network, they used a radio model. Besides, they assumed a fitness function with some fitness parameters such as direct distance to sink, cluster distance, transfer energy, and number of transmissions. The proposed algorithm maximizes the fitness function which is more energy efficient than a few existing cluster-based routing protocols in this work.

The problem of energy-efficient clustering in WSNs has been addressed by [10]. They proposed a novel clustering algorithm which improves the rate of data aggregation in the network. By ensuring that only nodes with a sufficient energy are selected as cluster-heads, they greatly decreased the consumed energy.[11]consider the distance for data transmission between sensor nodes that evaluates the fitness of a network. In their study, the actual energy consumption of the sensor nodes was varied depending on defining clusterheads and battery status of sensor nodes. 


\subsection{CLUSTERING IN WSN}

Sensor node are densely deployed in wireless sensor network that means physical environment would produce very similar data in close by sensor node and transmitting such type of data is more redundant. So all these issues give confidence in using some kind of grouping of sensor nodes such that group of sensor node can combine or compress data together and transmit only compact data to base station. This can reduce localized traffic in individual group and also reduce global data. This grouping process of sensor nodes in a densely deployed large scale sensor node is known as clustering. The method of combing data and compress data belonging to a single cluster called data aggregation.

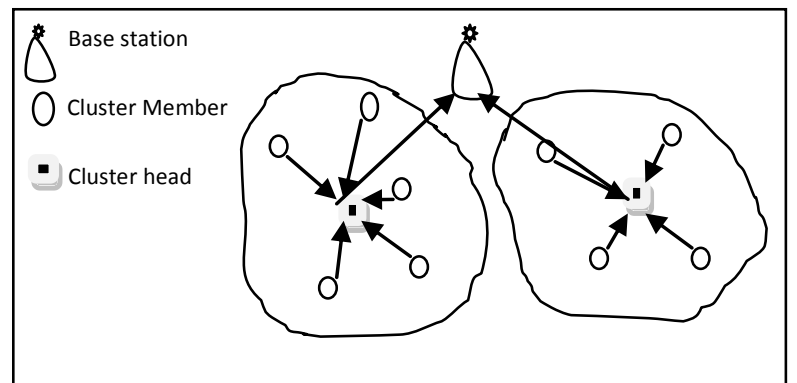

Fig 1: Cluster Based Data Aggregation in WSN

\subsection{VORONOI DIAGRAM}

Computational geometry is frequently used in WSN coverage optimization, the most commonly used computational geometry approach are Voronoi diagram and Delaunay triangulation [12]. Voronoi diagram is partition of cells in such a way that points inside a polygon are closer to that cell than any other cells thus one of the vertices of the Polygon is the farthest point of the polygon to the site inside it. Therefore Voronoi diagram can be used as one of the sampling method in determining WSN coverage; with the sensors act as the sites, if all Voronoi polygons vertices are covered then the field is fully covered otherwise coverage holes exist.

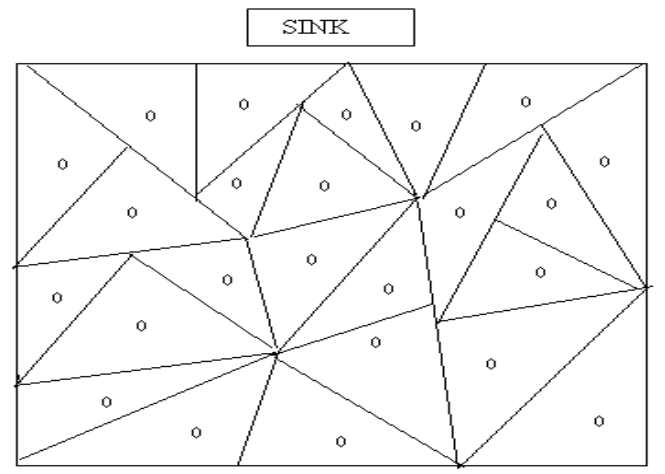

Fig. 2 Illustrates a Voronoi diagram

A Voronoi diagram for $\mathrm{N}$ sensors $\mathrm{s}_{1}, \mathrm{~s}_{2}, \ldots, \mathrm{s}_{\mathrm{N}}$ in a given plane is defined as the subdivision of the plane into $\mathrm{N}$ cells with one sensor, such that the distance between any point in a cell and the sensor of the cell is closer than that distance between this point and any other sensors. A Voronoi diagram is initially built up for all stationary sensor nodes, assuming that each node knows its own and its neighbor's coordinates. After the local Voronoi diagram construction, the sensor field is divided into sub regions of Voronoi cells and each stationary node is within a Voronoi cell. A node is a Voronoi neighbor of another one if they share a Voronoi edge. Fig.2 illustrates a Voronoi diagram in a bounded sensor field, where the boundaries of the sensor field contribute to a Voronoi cell. ask that authors follow some simple guidelines. In essence, we ask you to make your paper look exactly like this document. The easiest way to do this is simply to download the template, and replace the content with your own material.

\section{PROBLEM STATEMENT}

In this work, consider a sensor network application where each sensor is capable of aggregating multiple incoming data packets with its own data and forwards a single data packet of fixed length. Each node is equipped with small amount of energy and the objective is to keep all sensors working as long as possible in order to expand the life time of WSN.

Definition 1 In a data gathering epoch, base station accepts data from all nodes in the field. Each sensor acquires the required data samples form its environment, aggregates any incoming packets from its neighbors, and forwards the aggregated packet to its parent or base station.

Definition 2 The network lifetime is defined as the number of rounds until all nodes are in a working condition.

Definition 3 The load of a sensor node is defined as the energy required to receive all the incoming data packets from its children and to transmit the aggregated data to its parent.

\section{METHODOLOGY}

In proposed VBGC, it is assumed that all sensors are of the same type, deployed randomly in the sensing field. At first proposed algorithm uses voronoi diagram to find each sensor's coverage region by dividing given area into cells and then employs data mining process like clustering and data aggregation. Genetic clustering algorithm which is proposed for grouping optimal number of sensor nodes into clusters. In each $\mathrm{CH}$, data aggregation function is performed such as MIN,MAX, AVG so as to minimize the energy spent by each node.

Let assume that there are $\mathrm{n}$ sensors, $\mathrm{S} 1, \mathrm{~S} 2, \ldots, \mathrm{Sn}$, randomly deployed in field to detect its region R. Each sensor is able detect small range of region surrounding it (i.e) $r$, and connect to all those neighbors within its transmission range. The proposed methodology can be divided into four steps. The first step is to location discovery, which is used to determine the position of each sensor. The second step is detection range partition, where each sensor uses Voronoi diagram algorithm to delimit its responsible detection range. The third step is Genetic Algorithm for clustering and the final step is data aggregation.

\subsection{Location Discovery}

The first step is to determine the position of each sensor. If each sensor is equipped with a GPS, the system could have the absolute position of each sensor. In the proposed method sensor are considered to be static and location or position of the sensor's are known in prior by the base station through broadcasting of sensor's position.

\subsection{Detection Range Partition Using Voronoi}

After the first step, each sensor will be able to know the relative positions of its 1-hop neighbors. The next step is to determine the responsible detection range of each sensor. In 
this research, utilization of Voronoi diagram is to delimit the responsible detection range of each sensor. Voronoi diagram can be used to divide an area into sub-areas. In a Voronoi diagram, it holds the property that the nearest site of any point $\mathrm{x}$ in a sub-area $\mathrm{V}(\mathrm{Pi})$ must be $\mathrm{Pi}$ (site).

$$
\begin{aligned}
& \text { Let } \mathrm{P}=\left\{\boldsymbol{P}_{1}, \boldsymbol{P}_{2}, \ldots, \boldsymbol{P}_{n}\right\}, n \geq 2, \mathrm{P} \text { is a set of nodes in an } \\
& \text { area, and } \boldsymbol{P}_{1}, \boldsymbol{P}_{2}, \ldots, \boldsymbol{P}_{n} \text { are sites } \\
& V(P)=\left\{x: \boldsymbol{P}_{i}-x \leq \boldsymbol{P}_{j}-x, \forall j \neq i\right\} \\
& V(P)=\left\{V\left(P_{1}\right), V\left(P_{2}\right), \ldots . V\left(P_{n}\right)\right\} \\
& V(P) \text { is called a voronoi diagram }
\end{aligned}
$$

\section{PROPOSED VBGC}

The wireless sensor nodes are deployed in the sensing field $\mathrm{R}$ and each sensor know its position by using location discovery method. Then construct voronoi diagram for the given field using the equations in Table 1 in order cover the entire region R. Subsequently the genetic clustering algorithm is used to cluster the nodes in the field. Fitness function of GA is measured as Euclidian distance of sensor nodes and residual energy of each sensor. Using table 2, from the step1 to step 6 optimal cluster head $(\mathrm{CH})$ are elected and optimal number clusters are formed. Once the clusters are obtained sensed data in each node is transmitted from it to its cluster head $(\mathrm{CH})$. Finally data aggregation is carried out in each $\mathrm{CH}$, the cluster head will transmits only the aggregate information to the base station which is useful for further decision making process to the end user. Data aggregation is done so as to limit the energy spent by each node which in turn increase the total network life time.

$$
\begin{aligned}
& \text { Step 1. Generate initial population by means of V(P) } \\
& \text { Step 2. Evaluate fitness of all the individuals in the population } \\
& \text { Step 3. Cluster the population according to the fitness value } \\
& \text { i. Randomly generate k cluster centers }(\mathrm{CH}) \\
& D=\left(d_{1}, d_{2}, \ldots, d_{i}\right)
\end{aligned}
$$

Step 9. Perform data aggregation in each $\mathrm{CH}$, using aggregate function such as 1. Avg 2. Max 3. Min

Select option

$$
\begin{aligned}
& \text { If (1) then compute } a v g=\frac{\sum_{n-1}^{n}\left(d_{i}\right)}{N} \\
& \text { If(2) then compute } \max =\max \left(d_{i}\right) \\
& \text { If(3) then compute } \min =\min \left(d_{i}\right)
\end{aligned}
$$

Fig 4. Voronoi Based Genetic Clustering algorithm

\section{SIMULATION AND SUMMARY}

\section{Energy calculation}

The sensor nodes are randomly deployed in the $100 \times 100$ meters and the base station is located out the sensing field. 
The number of times that nodes transmit their messages to the cluster heads and cluster heads transmit the message to the base station from the beginning of the network life is defined as number of transmissions and is employed as a scale to compare the network lifetime and different clustering methods. By the way the network will be alive until ten percents of the nodes are alive. Wireless channel is assumed to be ideal and so there is no retransmission of control packets because of collision. We used a radio setting model the same as the one in Heinzelman [4]. In our simulation we assumed that the $\mathrm{n}=4000 \mathrm{bits}, \mathrm{E}_{\text {elec }}=50 \mathrm{~nJ} / \mathrm{bit}$ and $\varepsilon=10 \mathrm{pJ} / \mathrm{bit} / \mathrm{m} 2$ and table 1 and 2. The radio model is followed as in [4], etc...

The transmitting energy is calculated by the equation,

$$
E_{T}(n, d)=n \times E_{\text {elec }}+n \times \varepsilon \times d^{2}
$$

Where $n=$ number of bits

$$
\begin{aligned}
& d=\text { distance between the nodes } \\
& E_{\text {elec }}=\text { radio energy dissipation and } \\
& \varepsilon=\text { free space dissipation energy. }
\end{aligned}
$$

The energy required to receive k-bits is,

$$
E_{R}(n)=E_{\text {elec }} \times n
$$

Data aggregation energy is,

$$
E_{A G}=5 n J / b i t
$$

\begin{tabular}{|l|l|}
\hline Parameter & Value \\
\hline Number of nodes & 100 \\
\hline Network size & $100^{*} 100 \mathrm{~m} 2$ \\
\hline Initial node energy & $2 \mathrm{j}$ \\
\hline Minimum energy & $0.0001 \mathrm{j}$ \\
\hline Network threshold & 0.1 of nodes be alive \\
\hline Data transfer rate & Band width=1 Mbps \\
\hline
\end{tabular}

Table 1: Network model

\begin{tabular}{|l|l|}
\hline Parameter & Value \\
\hline Population size & 100 \\
\hline Number of generation & 20 \\
\hline Mutation rate & 0.006 \\
\hline Crossover rate & 0.8 \\
\hline tournament selection & probability of 0.9 \\
\hline
\end{tabular}

Table 2.Genetic parameter

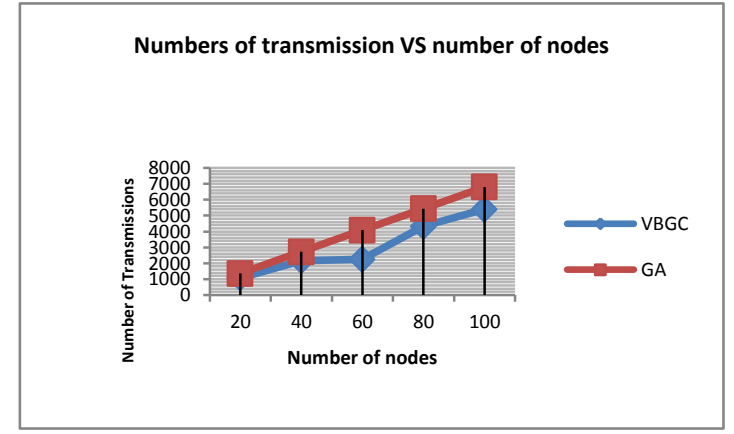

Fig 5: Number of transmissions VS number of node

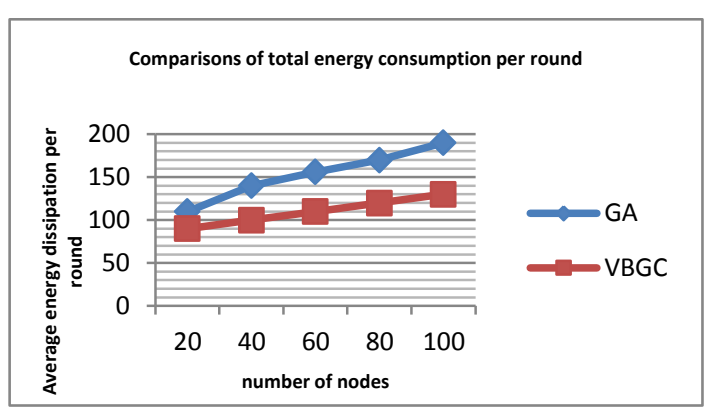

Fig 6: Average energy dissipation per round VS number of nodes

Fig. 5 and 6 shows the comparisons of total energy consumptions of proposed method per round Vs GA. GA has less information exchange than VBGC, therefore VBGC has the best energy consumption performance. As the number transmission increases performance of node decreases so if we increase the number of nodes then network life time will be extend and energy spent per node will be decreased. Hence the VBGC is efficient than any GA by reducing number of transmission to Basetion.

\section{CONCLUSION}

In this paper, energy efficient data aggregation using Voronoi Based Genetic Clustering algorithm were proposed for reducing the number of transmission from Cluster member to $\mathrm{CH}, \mathrm{CH}$ to base station and to increase the WSN life time. The voronoi diagram in VBGC was employed to determine the sensing range of each sensor since the total energy consumption is closely concerned with the number of cluster-heads and their position. Once the voronoi diagram is applied GA was applied for grouping the sensor node ,here proposed work tried to generate an optimal number of clusterheads and optimize the number of cluster members of each cluster-heads for data aggregation process. The Euclidian distance between sensor node and $\mathrm{CH}$ was considered to evaluate the fitness function of a genetic algorithm in a network. By this function, proposed method minimized the cost of transmission in this network. Data mining process further reduces the numbers data transmission from $\mathrm{CH}$ to sink node by data aggregation function such as MIN,MAX,AVG as additional benefit to clustering in network .To validate the algorithm, simulations had been carried out using MATLAB. Simulation results shows better performance of VBGC as compared to basic GA in terms of performance metrics like number of transmission, coverage area and total energy dissipation in the system. 


\section{REFERENCES}

[1] I. F. Akyildiz, W. Su, Y. Sankarasubramaniam, and E. Cayirci, "Wireless sensor networks: A survey," Computer Networks, vol. 38, no.4, pp. 393-422, March 2002.

[2] W. Heinzelman, A. Chandrakasan, and $\mathrm{H}$. Balakrishnan,"Energy-efficient communication protocol for wireless microsensor networks," in Proc. of the 33rd Annual Hawaii International Conference on System Sciences, pp. 3005-3014, January 2000.

[3] A. Abbasi and M. Younis, "A survey on clustering algorithms for wireless sensor networks," Computer Communications, vol. 30, pp.2826-2841, October 2007.

[4] W. Heinzelman, A. Chandrakasan, and H. Balakrishnan, "An application-specific protocol architecture for wireless microsensor networks," IEEE Trans. on Wireless Communications, 2002, 1(4), pp.660-670.

[5] O Younis and S Fahmy, "HEED: A hybrid, energyefficient,distributed clustering approach for ad hoc sensor networks," IEEE Transactions on Mobile Computing, vol. 3, pp. 366-379, 2004.

[6] D. E. Goldberg, Genetic Algorithms in Search, Optimization, and Machine Learning,Addison Wesley, Reading, MA, 1989.

[7] K. Sastry, D. Goldberg, and G. Kendall, Genetic Algorithms. Chapter 4 of Introductory Tutorials in Optimization and Decision Support Techniques, (eds. E. Burke and G. Kendall), pp. 97-125, Kluwer, 2005.

[8] R. Khanna, H. Liu, and H. H. Chen, "Self-organization of sensor networks using genetic algorithm," IEEE ICC '06, pp.3377-3382,June 2006.

[9] O. Islam, S. Hussain, and H. Zhang, "Genetic algorithm for nergy efficient clusters in wireless sensor networks," IEEE ITNG '07, pp. 147 - 154, 2007.
[10] J. Zhang, Y. Lin, C. Zhou, and J. Ouyang, "Optimal model for energy-efficient clustering in wireless sensor networks using global simulated annealing genetic algorithm,” IEEE IITAW ‘8, pp. 656-660, 2008.

[11] H. Seo, S. Oh, and C. Lee, "Evolutionary genetic algorithm for efficient clustering of wireless sensor networks," IEEE CCNC 2009,pp. 1 - 5, 2009

[12] Naeim Rahmani, Farhad Nematy et al" Node Placement for Maximum Coverage Based on Voronoi Diagram Using Genetic Algorithm in Wireless Sensor Networks", Australian Journal of Basic and Applied Sciences, 5(12): 3221-3232, 2011

[13] S.Nithya kalyani and S.Suresh kumar “ Optimal clustering Algorithm for Energy Efficient data Aggregation in WSN" European Journal of Scientific Research, ISSN 1450-216X Vol.78 No.1, ), pp.146155,2012 .

\section{BIOGRAPHICAL NOTES}

Mrs.S.Nithyakalyani received Bachelor of Engineering degree in Information Technology from Avinashiligam Deemed Universiry in 2004, and Master of Engineering in Computer Science and Engineering from Anna University in 2006. Currently she is working towards Ph.D degree in Anna University. Her area of interest includes in Data Mining, Network security. She is the life member of ISTE

Dr. S Suresh Kumar received M.S Degree from Birla Institute of Technology and Science in the year 1993, M.Tech from Indian Institute of Technology, Kharagpur in the year 2000.He has completed his Ph.D in the year 2009 from Anna University, Chennai .Presently he is the Principal of Vivekanandha College of Technology for Women. His area of interest includes Image Processing, Computer Network, Data mining, and Software Engineering. He published several paper in National and international Conferences and Journals. $\mathrm{He}$ is a life member of IEEE, ISTE, CSI. 